\title{
A Study on Singing Techniques of Sheng and Dan in Chinese Qiong Opera
}

\author{
Hong Zheng ${ }^{1}$, Xiuqing Fang ${ }^{2}$ \\ ${ }^{1}$ Associate Professor, Department of vocal music and opera, School of Music and Dance, Hainan \\ University, China, cjm95588@163.com \\ ${ }^{2}$ Adjunct Professor, Media \& Arts Management, Sogang University, Korea, artist@sogang.ac.kr \\ Corresponding author: Xiuqing Fang
}

\begin{abstract}
The Qiong opera has been a traditional drama in China for 300 years. In June 2008, Qiong opera was approved by the state council of the People's Republic of China to be included in the second batch on the list of National Intangible Cultural Heritage. However, with the rapid development of material culture, Qiong opera has been declining. One of the important reasons was unscientific vocalization. Taking the "Sheng" and "Dan" of five role types in Qiong opera as examples, this paper adopted the survey interview method and comparative analysis method. First, the survey interview method was adopted. Through the study of 5 troupes: Joan theater Hainan Province, Haikou City Qiong Opera Performing Arts Co. Ltd, Wenchang Qiong Opera Troupe, Qionghai Qiong Opera Troupe, Chengmai Qiong Opera Troupe, and 30 professional actors, the researchers obtained the primary information, studied vocalizing characteristics of Qiong opera, and pointed out the weakness of Qiong opera actors. At the same time, the students were trained of "Qiong Opera Talent Cultivation" project in bel canto vocal skills such as breathing, resonance, and articulation, and achieved remarkable results. Then comparative analysis method was adopted to compare and analyze the students' vocalizing conditions before and after taking bel canto vocalization classes. At the same time, the Qiong opera vocalization techniques were compared with bel canto, putting forward the thought that we could draw lessons from breathing, throat states, resonance, and articulation skills in bel canto to improve Qiong opera singing. The study showed that there was not a scientific vocalizing method in Qiong opera. Sheng had three technical problems when singing. First of all, the voice was not slack enough, and most of the actors had high throat positions. Next, the tone of the middle area was not clear, and it was difficult to sing in treble. Finally, resonance was not used properly. Dan also had three main problems. First, the improper mixing of true voice and falsetto in the mid-voice area and falsetto was comparatively overused. Second, the overuse of the throat muscles and poor vocal cord closure led to tightening, squeezing, and pinching voice. Finally, the pronunciation and articulation were not clear. Lessons could be drawn from bel canto on four aspects: first, chest and abdominal breathing method that made deep and coherent breaths; second, throat states in bel canto; third, the mixed resonance of bel canto; fourth, the articulation skills in bel canto.
\end{abstract}

Keywords: Opera, Qiong Opera, Vocalization Technique, Roles, Singing

\section{Introduction}

\subsection{Background and Objectives}

Received: May 21, 2021; $1^{\text {st }}$ Review Result: July 07, 2021; $2^{\text {nd }}$ Review Result: August 23, 2021 Accepted: September 30, 2021 
The history of Qiong opera can be traced back to the middle period of the Ming Dynasty in China. The modern Qiong opera was formed after the integration of the traditional drama Yiyang tune of Jiangxi Province and the local folk tune of Hainan province[1]. Qiong opera is sung in Hainan dialect, and it includes five role types which are Sheng(the male), Dan(the female), Jing(the painted face), Mo(the aged), and Chou(the clown). Performers use the skills of song, speech, dance, and combat in movements, among which, song is the most important. Different characters have a complete set of performing skills, makeups, and costumes. Song as a skill is an important channel to express characteristics and personality, which can effectively improve the artistic performance effect of operas and deepen the impression of the public[2]. It is very necessary to analyze the vocalization techniques of different professions in Qiong opera, because the performing artists of Qiong opera are all famous for their excellent singing.

The vocalization of Qiong opera lacks scientific theoretical guidance. Song, as one of the most important means to express characters' thoughts and emotions, plays a vital role in the development of drama. The singing of bel canto is consistent with the laws of human vocalization, which guarantees the scientific vocalization of bel canto and greatly reduces the load of vocal cords. And there is a set of scientific teaching methods in bel canto that can solve all kinds of problems and achieve the great and scientific voice. Both Qiong opera and bel canto fully developed and used human voice, thus forming a relatively complete art form, so they had certain intercommunity. The research aims to fill the gap in the field of applying bel canto singing techniques to Qiong opera, improve the singing ability of actors, promote the inheritance and development of Qiong opera, and accelerate the prosperity of cultural undertakings in Hainan Free Trade Port of China.

\subsection{Literature Review}

There are many studies on the development history and inheritance of Qiong Opera. Hainan Qiong Opera History written by Xing Jiyuan mainly told the origin, development history and discussion on the future development of Qiong Opera[3]. Zhao Kangtai's On The Culture of Qiong Opera expounded the relationship between Qiong opera and Hainan culture[4]. However, there was seldom systematic and targeted research on actors' vocalization technology. Wang Guonie's the Analysis about the Classification of Qiong Opera Singing and Art Characteristics analyzed the vocalizing characteristics of Qiong opera[5], Xie Chengju's The Re-cognition and Thinking on Qiong Opera Singing Reform and Characteristics only emphasized the necessity of reforming the Qiong opera vocalization[6]. There are very few papers that study the vocalization that based on scientific singing techniques of bel canto, therefore, the research direction of this paper is innovative and is necessary in academic research.

\section{Research Methods}

In most plays of Qiong opera, "Sheng" and "Dan" play a major role in the whole cast team, and the actors have a large number of arias. Therefore, the analysis of their singing methods is crucial to duet. To further understand the Qiong opera performances' vocalizing conditions, this paper adopted survey interview method to interview a total of 30 students of the Qiong Opera Talent Cultivation project sponsored by China National Arts Fund in 2018, of whom 20 played Dan roles, and 10 played Sheng roles. Ages from 19 to 35 years old can be said to be their best age. The actors were supposed to have great timbre and stable voice conditions, but in addition to a few performers with good voice conditions, the voice of the rest still had obvious technical problems of vocalization, especially the performers in remote areas. Such as two actresses in Dingan opera troupe, they obviously used too much falsetto, resulting in small sound volume and tight, screechy voice. Voices like this can hardly 
finish their characters' arias. Long-term inappropriate use of the voice led to various voice diseases, such as hoarseness and serious throat inflammation, which were extremely harmful to actors. Comparative analysis method was adopted to compare and analyze students' vocalizing conditions before and after taking bel canto vocalization classes. The students of "the Qiong Opera Talent Cultivation" project have initially learned correct sound and aesthetic concepts within two months, mastered the principle of breathing and could reasonably use resonance, and some of them who have tight, squeezed, and unstable voices have improved a lot. After two months of training, we made a report-back performance in Joan Theater Hainan Province. Nearly 500 professional and amateur Qiong opera actors gathered in the theater. In the performance, students sang classical opera pieces from 12 different plays. The progress of actors was significant, their voices sounded much gentler, and the phenomena of irregular shaking, tight, squeezed and pinched voices have been improved. The performance received unanimous praise from the audience.

This study was carried out from three aspects. One was to investigate and study the vocalization of some troupe actors, so as to learn the weakness of Qiong opera's vocalization. Second, compared Qiong opera and bel canto vocalization, and studied the necessity for Qiong opera to draw lessons from bel canto. The third was to make a comparative study on the vocalization of students before and after training, and to prove the feasibility of applying bel canto vocalizing techniques to Qiong opera through practice.

\section{Present Situation of Qiong Opera Performers' Vocalization}

The charm of Qiong opera lies in interpreting the story through actors' singing, reading, and acting, and the performances should be beautiful, euphonious, and moving. Actors shape the characters and expression feelings by traditional skills of song, speech, dance, and combat. It follows that singing in Qiong opera is a vital and an indispensable part. The Chinese language is polysyllabic and makes the sounds of Qiong opera complex and changeable, causing pressure on mouths, jaws, throats and vocal cords of Qiong opera actors. The survey interview method was adopted. Through the study of 5 troupes: Joan theater Hainan Province, Haikou City Qiong Opera Performing Arts Co. Ltd, Wenchang Qiong Opera Troupe, Qionghai Qiong Opera Troupe, Chengmai Qiong Opera Troupe, we obtained the primary information. By observing the singing techniques of Sheng and Dan in various professional performances, it could be found that the singing difficulties of Qiong opera performers generally include three aspects. First, the use of true voice and falsetto. Qiong opera actors could not achieve the natural transition between their true voice and falsetto. In particular, many modern composers tried to express mood by deliberately widening the vocal range of the melody in their works, which made it difficult for many actors to rely on their true voice to sing. After watching the video and listening to the recordings, it could be found that the actors sang only in their true voice, which caused dry and stiff tone and the lack of overtones. It was relatively easy for actors to sing in the lower register but difficult to struggle with their vocal cords in the higher register. In addition, the long-term rehearsal and performance of Qiong opera easily led to vocal cords fatigue of actors, especially when they used only their true voices to sing. If a play lasted for too long, the actor's throat was bound to swell with blood and water. However, Qiong opera often rehearsed and performed for several days without interruption. The performance of a play usually lasted for two to five hours, sometimes twenty performances in a month during the peak season, which caused actors to be in the state of long-term fatigue and difficult to continue their singing[7]. Only by learning and improving the singing methods to achieve the natural transition between the true and falsetto can the actors easily reach high pitch and keep pure and mellow timbre. The second was resonance. Actors should learn vocal skills and appropriately use head cavity, oral cavity, and chest cavity resonances to sing. The third was the voice conditions of performers. A good voice referred not only to actor's innate condition, but also to a voice 
that has been improved through hard work[8]. One's voice can be loud and mellow after long-term professional vocal training. All in all, problems caused by improper use of true voice and falsetto included: difficulty in using bass and treble; difficulty in producing soft sounds; lack of resonance due to ambiguous articulation; difficulty in shifting tones flexibly; obvious trace of conversion between high and low voice and so on. Only by addressing these vocal barriers can actors make better use of their voices, ensure the natural flow of their voice, show outstanding expressive ability, and better shape the characters [9]. [Table 1] below is the number of performers with vocalization problems in the troupes investigated by the author. It can be seen that many actors have various technical problems in vocalization.

[Table 1] Statistics of Performers' Voice Conditions from Some Hainan Troupes (unit: Person)

\begin{tabular}{|c|c|c|c|c|c|c|c|c|c|c|}
\hline \multirow[t]{2}{*}{ Voice } & \multicolumn{2}{|c|}{$\begin{array}{l}\text { Joan theater } \\
\text { Hainan Province }\end{array}$} & \multicolumn{2}{|c|}{$\begin{array}{c}\text { Haikou City } \\
\text { Qiong Opera } \\
\text { Performing Arts } \\
\text { Co. Ltd }\end{array}$} & \multicolumn{2}{|c|}{$\begin{array}{c}\text { Wenchang Qiong } \\
\text { Opera Troupe }\end{array}$} & \multicolumn{2}{|c|}{$\begin{array}{c}\text { Qionghai Qiong } \\
\text { Opera Troupe }\end{array}$} & \multicolumn{2}{|c|}{$\begin{array}{c}\text { Chengmai Qiong } \\
\text { Opera Troupe }\end{array}$} \\
\hline & Number & Ratio & Number & Ratio & Number & Ratio & Number & Ratio & Number & Ratio \\
\hline $\begin{array}{l}\text { Overused } \\
\text { Falsetto }\end{array}$ & 8 & $25 \%$ & 7 & $23 \%$ & 10 & $45 \%$ & 12 & $63 \%$ & 11 & $52 \%$ \\
\hline $\begin{array}{l}\text { Inappropriate } \\
\text { Resonance }\end{array}$ & 7 & $22 \%$ & 6 & $20 \%$ & 8 & $36 \%$ & 11 & $58 \%$ & 9 & $43 \%$ \\
\hline $\begin{array}{c}\text { Squeezing and } \\
\text { Tight Voice }\end{array}$ & 7 & $22 \%$ & 7 & $23 \%$ & 9 & $41 \%$ & 12 & $63 \%$ & 10 & $48 \%$ \\
\hline $\begin{array}{c}\text { Obscure } \\
\text { Articulation }\end{array}$ & 5 & $16 \%$ & 6 & $20 \%$ & 6 & $27 \%$ & 7 & $37 \%$ & 8 & $38 \%$ \\
\hline $\begin{array}{l}\text { Poor Vocal } \\
\text { Cord Closure }\end{array}$ & 7 & $22 \%$ & 6 & $20 \%$ & 8 & $36 \%$ & 7 & $37 \%$ & 8 & $38 \%$ \\
\hline Total Number & \multicolumn{2}{|c|}{32} & \multicolumn{2}{|c|}{30} & \multicolumn{2}{|c|}{22} & \multicolumn{2}{|c|}{19} & \multicolumn{2}{|c|}{21} \\
\hline
\end{tabular}

At present, Hainan has no high level Qiong opera school. Teachers are limited and the threshold for admission is low. Given that vocalization skills were very difficult courses, many actors' vocal training foundations were relatively weak. There were even actors who, without any professional training, simply imitated other actors' singing skills. It is well known that voice training changes with age. Moreover, the voice condition of individual is limited, thus the simple imitation is disadvantageous to voice protection. In addition, young actors generally lack vocal charm, mainly because they do not have their own mature and perfect vocalization techniques. Actors in Qiong opera need to achieve the organic unity of true voice and falsetto, to maintain clear articulation, to achieve sound transformation through the regulation of breath, and to make more use of abdominal breathing, so as to produce a brighter and more magnetic sound[10]. Therefore, it is necessary to continue to further the analysis and research on the vocal characteristics of Qiong opera to improve the vocal techniques of actors.

\section{Analysis on Voice of Sheng and Dan}

\subsection{About Sheng}

In the Qiong opera, Sheng mainly includes Zheng Sheng, Tie Sheng, Wu Sheng and Xiaohai Sheng. 
The Sheng in the early Qiong opera was generally referred to as Zheng Sheng, who had long black beard and was mostly the protagonist of the play. The male who did not dress up long and wore no black beard was called Xiaohai Sheng (young male) and was just a supporting actor in operas. With the declination of the black beard as decorations, Sheng no longer hung the beard in performances. Xiao Sheng are more common in Sheng, and mainly takes song and dance as the most important skills.

\subsection{Vocalizing Characteristics of Sheng}

When performing, the male characters in Qiong operas are expected to have a high-pitched, rounded voice and tend to be masculine. For example, for Zheng Sheng, the range should be broad and strong; the voice should have good lines, the use of breath should be smooth and not too high, the support of the diaphragm should always be maintained, the high voice should be sung to the bottom, and the low voice should be sung at the top of the voice, the breath and the vocal cords should be well balanced, and the enunciation should be clear. Hainan dialect is used in Qiong opera, so actors should retain the charm of dialect as far as possible. They should pay attention to the use of voice and maintain its smoothness. The resonance cavity was trained according to the actor's own voice condition[11]. Compared with Xiao Sheng, Wu Sheng's voice should have more tension, and their chest resonance can be moderately increased. In order to mold the role, the drama fragment which Wu Sheng performs has a big melody span. Most of the time they even have to sing during combat, which requires more control of breath and diaphragm. As a result, the actors need to be able to control their breath smoothly and keep their larynx steady during long-term training. Their voices are also more upright and masculine than Xiao Sheng's. The Xiaohai Sheng are played mainly by young men, so the voices of these actors need to have the energy and passion of adolescents, which are close to the timbre of children's voices. They have relatively light breaths and smaller bass width, and are able to hit the high notes more easily, which makes their voices more flexible and lyrical.

The voices of "Sheng" have a more obvious recognition, generally showing a natural, gentle tone, elegant and generous image[12]. Since ancient times, there have been many famous singers, most of whom were famous for their singing characteristics. For example, Tan Qicai, a young singer in Qiong opera, attached great importance to the control of words, sounds, qi and section, and followed the high and round voice of Sheng roles. Chen Juncai, known as the "King of Sheng in Qiong Opera", because of some deficiencies in the voice condition, sang in the tune board style and by tongue-enunciating, which is still very charming and meaningful. National first-class actor Chen Hua's singing has formed its own unique style, known as "Chen Hua Style", whose voice was like a small lyric tenor. It was very soft, with clear lines, bright, with tension, and recognizable. He played the classic roles of "Duan Yougong", "Lin Fangui", "Zhang Wenxiu" and "Gao Wenju" in classic plays like Zhang Wenxiu, Guangdong Kaike, and Lin Fangjia[13].

\subsection{About Dan}

In Qiong opera, Dan is mainly divided into Zheng Dan, Tie Dan, Hua Dan, Lao Dan, Cai Dan, Wu Dan, and MeiXiang Dan. Dan as female role is more elegant and gentler. Zheng Dan often plays elite and virtuous female roles. The early female Dan, such as Mo Aihua, Du Jianhong, Deng Wuhua, all sang with falsetto. Beginning in the 1920s, some singers switched to "true voice combined falsetto", such as Huang Xiaowen, Huang Yingrong, etc., while some artists used "true voice", such as Hong Mei, Lin Daoxiu, Li Guiqin, etc. In modern times, Dan roles often sing with a mixture of true voice and falsetto. 


\subsection{Voice Characteristics of Dan}

Among the seven kinds of Dan, Zheng Dan, Hua Dan, Lao Dan, and Mei XiangDan appear more. There are obvious differences between Zheng Dan and Lao Dan in the characteristics of singing voices. Zheng Dan's status in the drama is equivalent to that of the heroine in the movie. Their performances are the cores of presenting the characters in the whole play. In general, Zheng Dan's voice sounds more lyrical, which is similar to the lyric soprano in sound classification. The images are mostly positive figures such as young girls and gentle ladies, while Lao Dan are always the roles of mother and queen, so the expressions of voices differ from each other. No matter what kind of Dan roles, it is necessary to show the characteristics of the roles in the end, and combine the voice with emotion, express feelings through voice to give vivid performances. It requires the organic combination of sound, cavity, character, qi, to achieve the perfect combination of voice, emotion and the roles in the play.

It can be seen from [Table 2] below that there are obvious distinctions between Zheng Dan and Lao Dan's vocalizing characteristics, especially in their timbres, vocal ranges and resonances. In general, the vocal ranges of Zheng Dan are wider than those of Lao Dan, and their voices are brighter in soprano, while the bass tones of Lao Dan are relatively dim, and resonance cavities of Lao Dan are larger.

[Table 2] A Comparative Vocal Analysis of Zheng Dan and Lao Dan in Qiong Opera

\begin{tabular}{|c|c|c|}
\hline $\begin{array}{c}\text { Features of } \\
\text { Vocalizing } \\
\text { Techniques }\end{array}$ & Zheng Dan & Lao Dan \\
\hline Tone & $\begin{array}{c}\text { The vocal cords are thin and short, making it easy to reach } \\
\text { high notes. The tone is soft and beautiful, with strong } \\
\text { penetration and linearity. In modern repertoire, most Zheng } \\
\text { Dan actors sing through a mixture of true voice and } \\
\text { falsetto. }\end{array}$ & $\begin{array}{c}\text { The vocal cords are slightly longer, so } \\
\text { the sound is more penetrating with high } \\
\text { tension. The timbre is lower than that } \\
\text { of Zheng Dan, especially in a low tone. }\end{array}$ \\
\hline Breath & $\begin{array}{c}\text { The breath is flowing. The diaphragm is flexible and has a } \\
\text { good balance with the vocal cords. }\end{array}$ & $\begin{array}{c}\text { The diaphragm has strong elasticity and } \\
\text { support. }\end{array}$ \\
\hline Range & $\begin{array}{c}\text { The vocal range is wide and can reach 15 degrees after } \\
\text { training in general. }\end{array}$ & $\begin{array}{c}\text { The low and middle sound area is used } \\
\text { more and can be achieved by training } \\
\text { under normal circumstances. }\end{array}$ \\
\hline Articulation & $\begin{array}{c}\text { Hainan dialect is used for singing, which requires } \\
\text { articulation. }\end{array}$ & $\begin{array}{c}\text { Hainan dialect is used for singing, } \\
\text { which requires clear and strong } \\
\text { enunciation. }\end{array}$ \\
\hline Resonance & $\begin{array}{c}\text { The conditions of one's own voice. Most performers who } \\
\text { sing well use the resonance of the head cavity more in the } \\
\text { use the natural resonance in the middle voice region, and } \\
\text { add the chest resonance in the low voice region[14]. }\end{array}$ & $\begin{array}{c}\text { The resonance in the low vocal area is } \\
\text { arger, with chest resonance increasing, } \\
\text { and larger volume than that of Zheng } \\
\text { Dan. }\end{array}$ \\
\hline
\end{tabular}

Up to now, there are many classic roles in Qiong Opera, such as Jiang Yurui in Red Leaves Inscription, Wang Sanjie in Zhang Wenxiu, Yang Lizhen in One Hundred Years of Good Marriage, Meng Lijun in Meng Lijun, etc., which are all enduring roles in Qiong Opera[15]. Taking two Plum Blossom Prize winners Chen Suzhen, Lin Chuanmei as example, the roles that teacher Chen Suzhen played most in her life were Zheng Dan. She drew lessons from the mixture resonance of bel canto, changed the traditional opera thin timbre and solved the problems of obvious passaggio, singing 
sweetly and smoothly. Her main vocal characteristics were bright, clear, and charming. Teacher Lin Chuanmei's voice was broad and had rich tension. She could sing not only the role of young girls, such as Jiang Yurui in the Red Leaves but also the role of pantaloon, such as Lady Xian in Lady Xian. She was very flexible in the use of the voice of the Zheng Dan and Lao Dan, and she had a mellow and powerful voice that could accurately express the characters she played. In terms of voice, it can be heard that she drew lessons from bel canto vocalizing techniques such as breathing, resonance and articulation.

\section{Technical Problems on Vocalization of Sheng and Dan}

\subsection{Technical Problems on Vocalization of Sheng}

First, the larynx was high and unstable, which was a common problem in the initial stage of vocalization. The position of larynx is easy to rise with the rise of pitch, and it is more difficult to sing in the high-pitched area, resulting in a voice that lacks a sense of space. And the throat would feel pretty tired after performances. Such performances would bring a great load on vocal cords.

Second, the timbre was impure. In addition to the excessive use of the throat for a long time, the wrong way to produce sound can also make the tone in mid-vocal area not clean enough. Those with severe murmurs in the mid-vocal area were likely to have severe vocal cord injury or vocal cord disease[16]. Whether the vocal cords were damaged can be judged by whether there was mixed sound, even broken sound, whether it was difficult to reach high pitch and so on.

Third, the use of resonances was inappropriate. Resonance is the key to mellow voice. The timbre and volume of a good sound need to be achieved by adjusting the resonance. Most actors sing mainly with partial resonance, resulting in weak voices.

\subsection{Technical Problems on Vocalization of Dan}

First, excessive use of throat muscles and poor vocal cord closure resulted in tightening, squeezing, and pinching voice. Holding up throat to sing would cause tense muscles and poor pronunciation. The reason of poor vocal cord closure was that some students could not use breaths and vocal cords well that the voices were not concentrated and clear. However, excessive vocal cord closure was infeasible either, so it was necessary to find a balance.

Second, there was too much falsetto in the middle vocal area. Overused falsetto would result in poor sound tension, affecting the resonance, especially when singing across a large vocal range. The voice would not be loud and expressive enough.

Third, the articulation was obscure. The shape of the actor's mouth was mainly horizontal, and the mouth opening was small. The voice was mainly resonated at the front of the mouth, and the words uttered were relatively flat, sharp and tight. The timbre sound thin and the volume was small.

\subsection{Solutions to Problems on Vocalizing Techniques of Sheng}

First of all, solve the problem on vocalizing techniques. First, one should shape the concept. Students should be able to identify what sounds are produced when the larynx is high and how to judge the correct sounds. Second, begin throat vocalization exercises. Start with the vowel "a". First with a sigh to feel the throat by breathing resistance to continue to sound the feeling. Then gradually from "a" to "e", "i", "o", and "u". The actor should find the sound characteristic of each vowel as he practices it. For example, "o" and "u" tend to be lower at the back, while "e" and "i" are higher at the front. In practice they can be combined, such as singing "u" as "ue" and "i" as "io". In general, the 
practice of combining open and closed sounds makes up for the problem of misplacement caused by previous omissions, which makes the sound more balanced. Third, it is important to know that a high larynx position is also associated with breathing habits. Some people breathed loudly, but did not actually inhale at all. Their breaths were drawn to the mouth, but not to the diaphragm down, which was one of the reasons for the high laryngeal position. Fourth, the placement of larynx and chest sound fulcrum should be linked appropriately. Wait until the larynx is relaxed before making a sound.

Second, the key to solve this problem of impure timbre in middle vocal area lies in the vocalizing method. If the vocal cords are not severely damaged, it can be resolved with practice. First, the span of tone training should not be too large. It is better to start with a chromatic scale in the mid-range. Second, you should pay attention to the collocation of vocal cords and breath in practice. Keep the larynx stable and the voice high. Third, during the exercise, slowly remove the irregular impact of excess external forces on the vocal cords, such as throat muscles as well as breath holding and leakage, etc. When the whole sound converges in one direction, it gradually becomes clean and natural. Fourth, the natural stability of the middle vocal region is the basis of reaching the high pitch. When you hit a high note, keep your body excited and your facial muscles up.

Third, the solutions to the problem of improper resonance varies from person to person, but the most common one is to adjust the ratio of chest and head resonance. First, if you keep your voice loose when you sing, you can get a better resonance. When inspiriting, the lung inflates, two ribs open, and then the fulcrum of the chest and vocal cord breath produce resonance. The sound in this case is perfectly round and transparent. Therefore, the problem of chest resonance can be well solved. Second, the actor must make sure the mid-range sound remains stable and then rises slowly into the highpitched range. Under the cooperation of breath and vocal cords, a strong resonance effect will be produced. It seems like the voice is thrown through the head, and its track is shaped like a pyramid. That is to say, the bass is wide, and the treble is narrow. Therefore, the voice in the high range could become focused and loud.

\subsection{Solutions for Problems on Vocalizing Techniques of Dan}

First of all, solve the problems on overused falsetto. There should be body coordination exercises. To maintain the correct posture, the feet and body in the singing state should be relaxed. Actors need to hold their head high and do relaxation exercises, because only when the body relaxes the voice can be liberated and the breath can be relaxed. On one hand, students can practice with uttering the "do" sound, which requires the upper and lower lips to close slightly, creating a more regular sound against the airflow, and through this sound to control the breath and achieve balance. On the other hand, students can also use "si" sound to practice, which requires the upper and lower teeth slightly closed to generate resistance after the sound of "si". Through this sound, students can control the speed of the air flow and feel the balance of the body when controlling the air flow, and finally achieve the effect of relaxing the body and getting a smooth breath. Second, they should learn to distinguish the true voice and falsetto. If falsetto is used too much, students can find the true voice in a natural speaking voice. After the students are used to hearing their own true voice, they can find the true voice and falsetto voice by simple exercises in the middle voice area. They can only expand the voice range up and down by adjusting the sound in the middle zone well.

Second, to solve the problem of multi-glottal force, we must first solve the problem of vocal cord closure. Vocal cord closure should be moderately relaxed and tightened. When the vocalization is correct, the singer will feel relaxed, and the sound produced will also have a sense of beauty. The singer will get better sound effects if they keep practicing with this feeling. Second, the tight, squeezed, and pinched voice is caused by the irregular voice habit. Students could find the balance between 
breath and vocal cords in daily practice. Start with a soft voice, and let the vocal cords sound out slowly under the influence of the breath.

Third, solve the problem on obscure articulation. Qiong opera is sung in Hainan dialect, which has 18 initial consonants and 55 finals according to Hainan Wenchang dialect, which is a tough test of the actors' abilities to enunciate words. When pronouncing each word, actors should pay attention to put the rhyme in place and not to put too much stress on the starting consonants[17]. Actors of Dan should keep the enunciation to follow the trend of the voice line and keep the oral cavity small, otherwise it will cause slur enunciation. With the mixture of true voice and falsetto in the middle vocal area, the sound timbre will be very free and pleasant under the effect of breath, while in the low vocal region, the proportion of true voice can be added, like the sound of bass, to increase the resonance of the chest cavity, making the low vocal music more tense, powerful, and broader. The proportion of falsetto can be improved in the treble area.

\section{Implementation of Solving Problems on Sheng and Dan Vocalization Techniques}

In the process of training, comparative analysis method was adopted to solve the problem students' vocalizing techniques, and singing skills in bel canto were applied in Qiong opera. Through vocalizing training in classes and gradually targeted training with students' voice range and resonance, learning the characteristics of different ages and parts, students' voice characteristics and expressing abilities were explored in experiments. Starting from the middle voice area, and then gradually expanding the range, on the premise of not losing the charm of opera, the singing life expectancy and performances of Qiong opera performers could be improved through scientific vocalization method. After training of breathing, resonance, and articulation in bel canto singing, students in Qiong Opera Talent Cultivation program have made great progress. [Table 3] below is the training results of students of Qiong Opera Cultivation project. It can be seen that many students' vocalizing problems have been solved after the training.

[Table 3] The Training Results of Students of Qiong Opera Talent Cultivation Project (unit: Person)

\begin{tabular}{|c|c|c|c|c|c|c|}
\hline & \multicolumn{2}{|c|}{ Before Training } & \multicolumn{2}{c|}{ After Training } & \multicolumn{2}{c|}{ Reduced Number } \\
\hline Problems & Sheng & Dan & Sheng & Dan & Sheng & Dan \\
\hline Overused Falsetto & 5 & 13 & 3 & 5 & 2 & 8 \\
\hline Inappropriate Resonance & 6 & 7 & 2 & 3 & 4 & 4 \\
\hline Tight and Squeezing Voice & 6 & 12 & 3 & 4 & 3 & 8 \\
\hline Obscure Articulation & 4 & 6 & 2 & 2 & 2 & 4 \\
\hline Poor Vocal Cord Closure & 2 & 10 & 1 & 3 & 1 & 7 \\
\hline
\end{tabular}

From [Table 3] above, it can be seen that it is feasible to learn from singing techniques in bel canto. We could draw lessons from bel canto on four aspects: first, use chest and abdominal breathing method to make deep and coherent breaths. Second, throat states: open your throat, and let your larynx fall naturally. Third, the mixed resonance of bel canto, make the sound point in the chest. Fourth, the articulation skills, sing with the feeling of wide open mouth.

\section{Conclusion}

This paper analyzed the vocalization characteristics of Sheng and Dan in Qiong opera and the problems on vocalizing techniques through survey interview method and comparative analysis method, and put forward solutions and specific implementation plans. The study showed that the vocalization of Qiong opera has not yet formed a systematic, complete and detailed theory of singing and teaching. Sheng had three technical problems when singing. First of all, the voice was not slack enough, and 
most of the actors had high throat positions. Next, the tone of the middle area was not clear, and it was difficult to sing in treble. Finally, resonance was not used properly. Dan also had three main problems. First, falsetto was comparatively overused in the middle vocal area. Second, the overuse of the throat muscles and poor vocal cord closure led to tightening, squeezing, pinching voice. Finally, the pronunciation and articulation were not clear. To solve the existing technical problems of Sheng and Dan in Qiong opera, this paper put forward following solutions: First, explore the reforms of singing style of Qiong opera to win more audiences for Qiong opera and give it vitality. Second, start singing research classes and select students with developing potential to attend the classes.

The training results of students of Qiong Opera Talent Cultivation project showed that it was feasible to learn vocalizing techniques from bel canto. The lessons from bel canto could be drawn from four aspects: first, use chest and abdominal breathing method to make deep and coherent breaths, so as to produce mellow voice, concentrated and penetrating. Second, learn the throat states of bel canto. Open your throat, and let your larynx fall naturally. The lower larynx can not only make the breath smooth and deep, but also make full use of the resonance of singing, so as to improve singing ability. Larynx in the lower position of the throat state cannot only make the breath smooth and full, but also make full use of the resonance of singing, so as to improve singing ability. Third, use the mixed resonance of bel canto. Make the sound point in the chest, to ensure the use of chest resonance and head cavity resonance, with soft palate lifted and throat relaxed. With the voice flowing to the front, make the voice sound more mellow and confident. Fourth, learn the articulation skills of bel canto. Sing with the feeling of wide open mouth to make up for the deficiency of articulation in Qiong opera.

This research gave technical guidance to actors' singing techniques of Qiong opera, provided a broader vision for Qiong opera teaching, led actors to better serve Qiong opera through improving singing techniques, and made the singing of Qiong opera more natural and delicate. The limitation of this study was that it did not mention the physiology of the vocal organs and how they work in coordination. The next step is to learn from the physiological structure theory of singing organs in bel canto, study the coordination of various vocal organs, and establish a scientific and standardized vocalization theory of Qiong opera.

\section{References}

[1] K. Y. Fu, On the Intangible Cultural Heritage Value and Protection of Qiong Opera, Chongqing Normal University, Master's Thesis, (2019)

[2] K. Yuan, Research on the Yin School Singing Art of Yue Opera, Zhejiang Normal University, Master's Thesis, (2020)

[3] J. Y. Xing, Hainan Qiong Opera History, Hainan Publishing House, (2019)

[4] K. T. Zhao, On the Culture of Qiong Opera, Southern Publishing House, (2019)

[5] G. N. Wang, The Analysis about the Classification of Qiong Opera Singing and Art Characteristics, Charming China, (2021), No.19, pp.403-404, DOI: 10.12294/j.issn.1673-0992.2021.19.427

[6] C. J. Xie, The Re-cognition and Thinking on Qiong Opera Singing Reform and Characteristics, Collected Dramas, (2010), No.2, pp.69-74.

[7] C. M. Long, L. S. Xie, Study on the Classification and Artistic Characteristics of Qiong Opera, Art Criticism, (2020), No.23, pp.63-66.

[8] X. X. Li, Hainan Qiong Drama Culture into the Classroom of the Current Situation and Implementation Path Research, Hainan Normal University, Master's Thesis, (2019) 
[9] X. L. Duan, On the Popularization and Application of Scientific Vocalization in the Teaching of Beijing Opera, Arts Education, (2020), No.4, pp.113-116, DOI : 10.16737/j.cnki.rwtx81281190.2020.04.035

[10] Q. C. Han, A Study on the Vocalization of Chinese Opera: A Topic that Needs Urgent Attention, People's Music, (2014), No.7, pp.35-37.

[11] M. Conceição, L. Silva, E. Morgado, M. Cardoso, The Artistic and Scientific Connections between Music and Mathematics, International Journal of Art and Culture Technology, (2018), Vol.2, No.2, pp.51-62.

[12] J. Y. Zhang, On the Singing Structure and Artistic Features of Qiong Opera, North Music, (2019), Vol.39, No.13, pp.76-77, DOI : 10.3969/j.issn.1002-767X.2019.13.053

[13] S. Y. Chung, Korean Classical Poetry and Song Education in the Revised Literature Textbooks of 2019, Asia-pacific Journal of Convergent Research Interchange, (2019), Vol.5, No.4, pp.83-91.

[14] K. Kalpana, P. Saikrishna, Y. J. Chang, M. J. Tian, A Study on Ratio Analysis, International Journal of Smart Business and Technology, (2016), Vol.4, No.1, pp.5-14.

[15] H. J. Moon, S. Y. Ihm, QSoSA (Quad-Space of Stage Acting) Model for Systematic Space Analysis of Stage Acting Based on Hermann Schmitz's Concept of Space, Asia-pacific Journal of Convergent Research Interchange, (2020), Vol.6, No.1, pp.1-18.

[16] S. K. Ryu, A Field Study on the Acceptance Characteristics of Korean Drama by Thailand Viewers, Asia-pacific Journal of Convergent Research Interchange, (2020), Vol.6, No.4, pp.33-46.

[17] Q. Zhao, X. Q. Fang, Chinese Localization of Musicals Co-developed by Korea and China: A Case Study on the Musicals "Bachelor's Vegetable Store", "In the Mood for Sorrow", and "Rimbaud", Asia-pacific Journal of Convergent Research Interchange, (2020), Vol.6, No.9, pp.151-167. 